\title{
Evaluation of a Case of Inflammatory Breast Cancer with ${ }^{18}$ F-FDG PET/CT
}

\author{
Zaid Al-Faham ${ }^{1}$, Sayf Al-Katib ${ }^{1}$, Ishmael Jaiyesimi² ${ }^{2}$ and Sharukh Bhavnagri ${ }^{1}$ \\ ${ }^{1}$ Department of Diagnostic Radiology and Molecular Imaging, William Beaumont School of Medicine, Oakland University, Royal \\ Oak, Michigan; and ${ }^{2}$ Department of Hematology and Oncology, William Beaumont School of Medicine, Oakland University, \\ Royal Oak, Michigan
}

Inflammatory breast cancer is a rare and aggressive form of cancer characterized by dermal lymphatic invasion and tumor embolization resulting in erythema and edema. In many cases, by the time of diagnosis there is already distant metastasis. Mammography, sonography, CT, and MRI are usually performed for initial staging; however, PET/CT can also be used for initial staging as it offers additional diagnostic information.

Key Words: inflammatory breast cancer; IBC; PET/CT

J Nucl Med Technol 2015; 43:289-291

DOI: 10.2967/jnmt.114.148494

\section{$\mathbf{I}_{\mathbf{n}}$}

nflammatory breast cancer (IBC) is the most aggressive form of locally advanced breast cancer. Fortunately, it is rare and composes only $1 \%-6 \%$ of breast cancer cases (1). Pathologically, this type of cancer demonstrates highly angiogenic properties, which account for its early metastasis. The imaging and physical examination findings of IBC mimic those of inflammatory processes, and it is important to distinguish between these etiologies in a timely manner for proper management. Approximately 30\% of women have stage IV de novo disease at diagnosis, and PET/CT has high sensitivity in identifying the extent of disease (2).

In this case report, we present the imaging findings of IBC on PET/CT with MRI correlation. We discuss the role of PET/CT in the workup and management of IBC, including diagnosis, staging, and prediction of overall survival in patients undergoing chemotherapy.

\section{CASE REPORT}

A 67-y-old woman presented after an area of erythema in her right axillary region had progressed to involve her right

\footnotetext{
Received Sep. 14, 2014; revision accepted Mar. 25, 2015.

For correspondence or reprints contact: Zaid Al-Faham, William Beaumont Hospital, 3601 W. 13 Mile Rd., Royal Oak, Ml 48073.

E-mail: zaidfaham@gmail.com

Published online Nov. 19, 2015.

COPYRIGHT (c) 2015 by the Society of Nuclear Medicine and Molecular Imaging, Inc.
}

breast. Examination revealed diffuse erythema and edema of the right breast. A mammogram performed $1 \mathrm{y}$ previously had shown no evidence of malignancy. Skin punch biopsy was performed and yielded invasive carcinoma compatible with IBC.

The patient subsequently underwent T2-weighted, maximumintensity-projection, contrast-enhanced breast MRI, which showed asymmetric skin thickening of the right breast, breast distortion, and breast retraction (Fig. 1A). Increased T2 signal within the thickened skin was indicative of edema. A large, irregular parenchymal mass with peritumoral edema and spiculated margins was also noted in the central right breast. Subtracted postcontrast imaging demonstrated enhancement of the mass (Fig. 1B) and right axillary lymphadenopathy (Fig. 1C). Additionally, the overlying skin showed abnormal contrast enhancement due to involvement with malignancy.

PET/CT for initial staging was then performed. Sixty minutes after intravenous administration of $7.05 \mathrm{~Bq}(19.06 \mathrm{mCi})$ of ${ }^{18}$ F-FDG, sequential unenhanced CT and then PET images were acquired. The images showed an infiltrating mass with increased radiotracer activity within the right breast and extending into the axilla; quantification showed a maximum SUV of 8.1 (Fig. 2). Diffuse hypermetabolic skin thickening of the right breast corresponded to the abnormal findings on MRI. Moreover, multiple regional and distant ${ }^{18} \mathrm{~F}-\mathrm{FDG}-$ avid lymph nodes were discovered, including the right axillary nodes and an enlarged portocaval node (Fig. 3). These findings were consistent with IBC with regional and distant metastases. Percutaneous core biopsy confirmed the presence of metastasis to the portocaval node.

\section{DISCUSSION}

IBC is the most aggressive form of breast cancer and has poorer survival than non-IBC tumors (3). Imaging plays an important role in cases of IBC to detect intraparenchymal breast lesions, nodal involvement, and distant metastasis (3). Mammography and sonography are the recommended imaging modalities in the initial workup of IBC. However, MRI is also used in many cases to provide additional sensitivity for detection of breast parenchymal lesions. 
FIGURE 1. (A) T2-weighted MR image showing diffuse asymmetric skin thickening of medial right breast, breast distortion, breast retraction, and increased signal (arrow) within the thickened skin, compatible with edema. (B) Postcontrast MR image showing enhancement of skin (arrowhead) and of irregular breast parenchymal mass (arrow). (C) Subtracted postcontrast MR image showing abnormal enlarged right axillary lymph node (arrow).
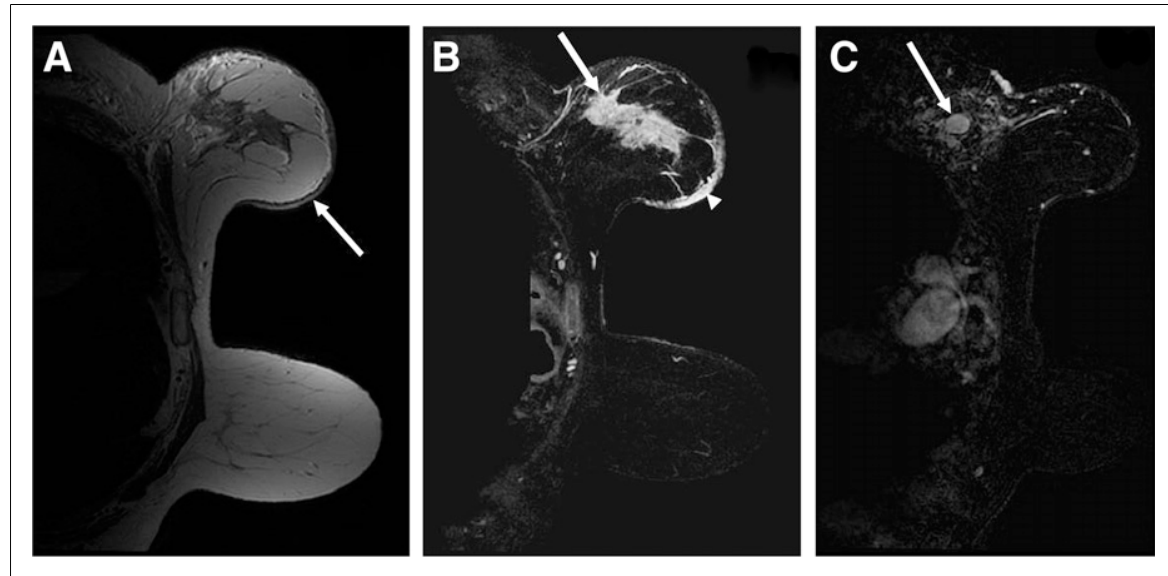

Although these imaging modalities provide excellent sensitivity for locoregional disease, additional imaging is required to establish the stage and extent of disease, which are critical for treatment planning. CT and bone scanning are still used for initial staging (3). However, the use of $\mathrm{PET} / \mathrm{CT}$ in this setting is increasing.

The usual findings in patients with IBC are multiple hypermetabolic breast foci associated with hypermetabolic thickened skin (1). PET/CT has high sensitivity for detection of breast lesions, axillary nodal involvement, and distant metastasis. Yang et al. reported that PET/CT had a sensitivity of $96 \%$ for the detection of breast parenchymal lesions, $95 \%$ for axillary metastasis, and $100 \%$ for distant metastasis (4). Champion et al. showed that PET/CT performed better than contrast-enhanced $\mathrm{CT}$ in the evaluation of regional lymph nodes (sensitivity, $93 \%$ vs. $86 \%$, and positive predictive value, $93 \%$ vs. $89 \%$ ) (2). In our patient, PET/CT was equal to breast MRI for the detection of locoregional disease, as both modalities detected the breast parenchymal lesion and the skin and axillary nodal involvement. PET/CT may give false-negative results in cases of small lesions, particularly in the liver and lung parenchyma. Also, false- positive results may occur in cases of inflammation or infection (5). Ipsilateral axillary lymph node uptake due to extravasation or partial subcutaneous injection of the antecubital region has been reported, thus giving false-positive results in some cases.

Multiple studies have shown that PET/CT detects metastases that were not previously suspected. For example, Carkaci et al. reported that among 41 patients with IBC, 7 of the 20 cases of distant metastases were not suspected before PET/CT (6). In our case, the staging PET/CT led to the discovery of distant nodal involvement in the portocaval region. PET/CT specifically is useful in detection of internal mammary lymph node involvement, which is predictive of recurrence (3).

In addition, PET/CT is useful for monitoring the response to treatment and for detecting early recurrence. Patients undergoing chemotherapy for IBC who show an interval decrease in tumor metabolic activity have better overall survival; every $10 \%$ decrease in SUV translates into a $15 \%$ lower probability of death, and complete resolution of tumor ${ }^{18} \mathrm{~F}-\mathrm{FDG}$ uptake translates into an $80 \%$ lower probability of death $(6)$.
FIGURE 2. PET/CT (top left), CT (bottom left), whole-body maximum-intensityprojection PET (top right), and axial PET (bottom right) images. Infiltrating mass with increased radiotracer activity within right breast extends into axilla.

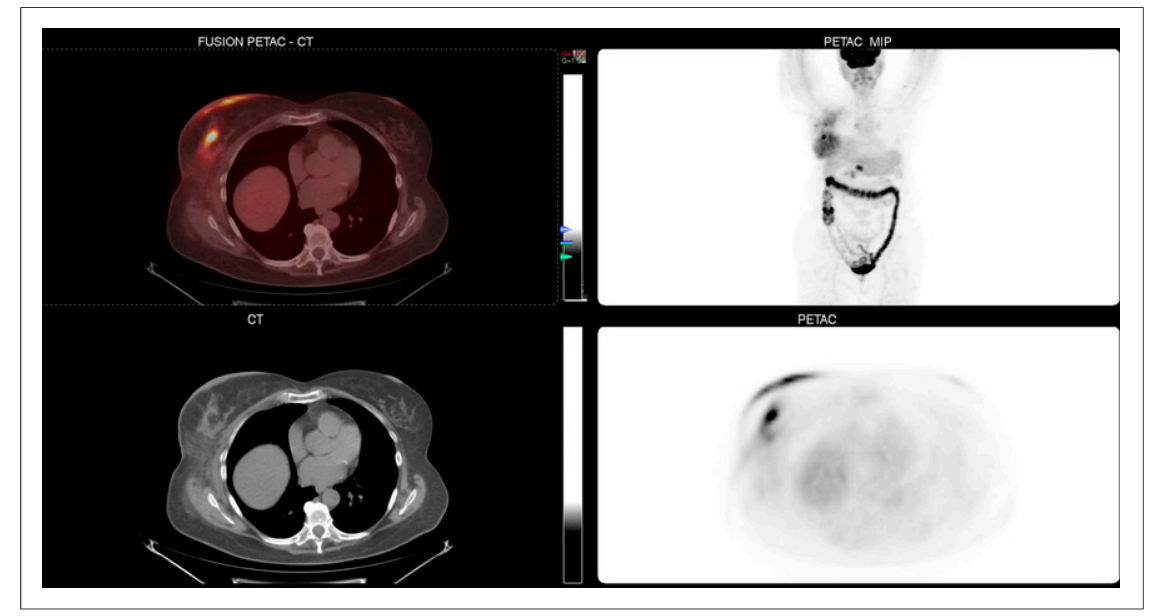




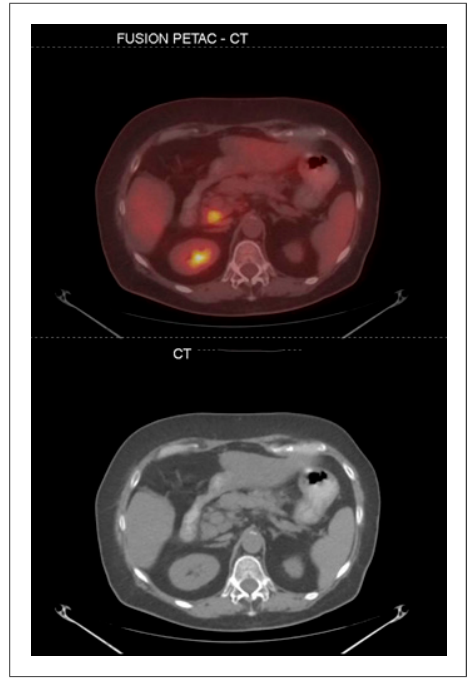

FIGURE 3. Axial PET/ CT (top) and CT (bottom) images of ${ }^{18} \mathrm{~F}-\mathrm{FDG}$-avid portocaval node.

In summary, MRI is more precise than mammography, sonography, and PET/CT for detecting cancer in an inflamed breast (6). PET/CT is useful for local and regional lymph node staging before neoadjuvant chemotherapy, for guidance regarding the fields to be irradiated after mastectomy, and for detection of remote metastases (5).

\section{CONCLUSION}

We have presented a case of IBC in which PET/CT was equal to MRI in evaluation of locoregional disease yet additionally provided detection of distant metastasis. PET/CT plays an important role in the diagnosis, staging, and monitoring of disease in IBC patients. Given the high likelihood of distant metastasis in cases of IBC, accurate initial staging with PET/CT is crucial as it affects treatment planning and ultimately patient outcome.

\section{DISCLOSURE}

No potential conflict of interest relevant to this article was reported.

\section{ACKNOWLEDGMENT}

We give special thanks to Robert Ceruti, CNMT, from the IT Department for his help.

\section{REFERENCES}

1. Alberini JL, Lerebours F, Wartski M, et al. ${ }^{18} \mathrm{~F}$-fluorodeoxyglucose positron emission tomography/computed tomography (FDG-PET/CT) imaging in the staging and prognosis of inflammatory breast cancer. Cancer. 2009;115: 5038-5047.

2. Champion L, Lerebours F, Cherel P, et al. ${ }^{18}$ F-FDG PET/CT imaging versus dynamic contrast-enhanced CT for staging and prognosis of inflammatory breast cancer. Eur J Nucl Med Mol Imaging. 2013;40:1206-1213.

3. Dawood S, Merajver SD, Viens P, et al. International expert panel on inflammatory breast cancer: consensus statement for standardized diagnosis and treatment. Ann Oncol. 2011;22:515-523.

4. Yang WT, Le-Petross HT, Macapinlac H, et al. Inflammatory breast cancer: PET/ CT, MRI, mammography, and sonography findings. Breast Cancer Res Treat. 2008;109:417-426.

5. de Bazelaire C, Groheux D, Chapellier M, et al. Breast inflammation: indications for MRI and PET-CT. Diagn Interv Imaging. 2012;93:104-115.

6. Carkaci S, Sherman CT, Ozkan E, et al. ${ }^{18}$ F-FDG PET/CT predicts survival in patients with inflammatory breast cancer undergoing neoadjuvant chemotherapy. Eur J Nucl Med Mol Imaging. 2013;40:1809-1816. 